\title{
Sum-Frequency Spectroscopic Studies of Ice Interfaces
}

\author{
Xing Wei*, Paulo B. Miranda ${ }^{\dagger}$, Chun Zhang, and Y. R. Shen \\ Department of Physics, University of California, and Materials Sciences Division, Lawrence \\ Berkeley National Laboratory, Berkeley, CA 94720
}

(December 11, 2001)

\begin{abstract}
Sum-frequency vibrational spectroscopy was used to study the (0001) surface of hexagonal ice $\left(\mathrm{I}_{\mathrm{h}}\right)$ in contact with: air, a hydrophobic substrate, and a hydrophilic substrate. The spectra in the $\mathrm{O}-\mathrm{H}$ stretch frequency range were obtained at various temperatures. For the air/ice interface, the degree of orientational order of the dangling $\mathrm{OH}$ bonds at the surface was measured as a function of temperature. Surface disordering appears to set in around $200 \mathrm{~K}$ and increases dramatically with temperature, which is a strong indication of the presence of surface melting of ice. For the hydrophobic and hydrophilic ice interfaces, a similar temperature dependence of the hydrogen-bonded $\mathrm{OH}$ stretch peak was observed. The free $\mathrm{OH}$ stretch mode, however, appears to be different from that of the air/ice interface due to the interactions with substrates at the interfaces.
\end{abstract}

PACS numbers: 64.70.Dv,68.35.Bs,42.62.Fi,42.65-k 


\section{INTRODUCTION}

To explain sintering of ice under relatively low pressure, Michael Faraday first proposed the existence of a liquid-like layer on an ice surface below the bulk melting temperature [1]. This is known today as surface melting or premelting of ice. Surface melting is actually a rather common behavior of many solids including lead [2,3], argon [4], germanium [5], gallium [6] and aluminum [7]. For ice, it is particularly interesting because of its many important consequences. It is believed to play a key role in phenomena such as reduction of the frictional coefficient of ice, electrification of thunder clouds, glacier flows, frost heaves, and ozone depletion $[8,9]$. The problem has been the subject of extensive theoretical and experimental investigations since 1968, when Fletcher first seriously considered the idea of Faraday [10].

The calculation of Fletcher based on electrostatic interactions [10] predicts that surface melting of ice is a complete surface disordering process (i.e., the quasi-liquid layer thickness diverges as the temperature approaches the bulk melting temperature), and the onset temperature is between -3 and $-6^{\circ} \mathrm{C}$. A more recent theory based on dispersive van der Waals interactions [11], however, predicts that the precess is incomplete (i.e., the quasi-liquid layer thickness remains finite before the bulk melting sets in). Molecular dynamics simulations of ice surface melting have also been carried out [12-14], and the reported onset temperature is as low as $-40^{\circ} \mathrm{C}[12]$. Today, a generally accepted theory of surface melting of ice has not yet been found.

Experimental investigation of surface melting of ice is not in any better shape either. Various techniques have been used to probe the phenomenon. They include photoemission [15], proton backscattering [16], ellipsometry [17,18], optical reflectometry and interference

microscopy [19], nuclear magnetic resonance [20,21], low energy electron diffraction [22], glancing angle x-ray diffraction [23], helium atom scattering [24], and atomic force microscopy $[25,26]$. Ice in contact with other solids has also been studied by wire regelation [27], quasi-elastic neutron scattering [28], and viscosity measurements [29]. While most of 
these experiments seem to have confirmed the existence of the liquid-like layer on the ice surface or interface, the details, such as the onset temperature and the temperature dependence of the layer thickness, vary widely from experiment to experiment. Presumably, different techniques measure different properties and liberal interpretations of the results could lead to large discrepancies. Today, the exact nature of surface melting of ice remains controversial, and the structure and properties of the quasi-liquid layer are far from being fully understood.

We note that one serious problem in the studies of ice surfeces is its incompatibility with ultrahigh vacuum (UHV) due to its high vapor pressure, which varies between $10^{-5}$ and 5 Torr in the temperature range of 175 to $273 \mathrm{~K}$ [30]. The high vapor pressure hinders the use of standard UHV surface science techniques at temperatures close to the ice melting temperature. Non-UHV techniques such as ellipsometry and x-ray diffraction can cover a wide range of temperatures, but do not have enough surface sensitivity.

Sum-frequency generation (SFG) vibrational spectroscopy [31] has recently been established as a powerful tool for probing surfaces and interfaces. It is highly surface-specific and sensitive, and does not require an ultra high vacuum environment. Therefore, it is ideally suited for studies of surfaces and interfaces of ice and water. It has been used before to investigate various interfaces of water [32-34], as well as an ice film grown on $\mathrm{Pt}(111)$ [35]. These experiments demonstrated the capability of SFG vibrational spectroscopy to yield structural information of water and ice that could not be obtained with other experimental techniques.

Here we report our studies of surface melting of hexagonal ice $\left(\mathrm{I}_{h}\right)$ using this technique. We focused on the ice interfaces of the (0001) orientation (basal plane), since the (0001) surface is most stable. Preliminary results on the air/ice interface have already been published [36]. They show that disordering of the (0001) surface becomes detectable at $200 \mathrm{~K}$, and increases as the temperature increases. A comparison of the results for the ice surface and for the supercooled water surface, however, reveals that the structures of the quasi-liquid layer on ice and the surface layer of liquid water are not the same. In this paper we present a more 
complete report of our work including a detailed description of the experimental preparation and results and the detailed analysis of the results. In addition, we also describe our SFG spectroscopic studies on fused quartz/ice interfaces with and without a hydrophobic silane monolayer.

\section{THEORETICAL BACKGROUND FOR SFG VIBRATIONAL SPECTROSCOPY ON ICE}

\section{A. Molecular orientation at surface}

The SFG vibrational spectroscopy technique has been described in earlier publications [31,37]. It has been shown that the SFG output in reflection from a surface excited by two overlapping input beams, one visible at $\omega_{1}$ and the other infrared at $\omega_{2}$, is proportional to the square of an effective surface nonlinear susceptibility, $\chi_{\text {eff }}^{(2)}\left(\omega_{s}=\omega_{1}+\omega_{2}\right)$, defined as

$$
\chi_{\mathrm{eff}}^{(2)}=\left[\mathbf{L}\left(\omega_{s}\right) \cdot \mathbf{e}_{s}\right] \cdot \chi^{(2)}:\left[\mathbf{L}\left(\omega_{1}\right) \cdot \mathbf{e}_{1}\right]\left[\mathbf{L}\left(\omega_{2}\right) \cdot \mathbf{e}_{2}\right]
$$

where $\mathbf{e}_{i}$ is a polarization unit vector of the optical field at $\omega_{i}, \mathbf{L}\left(\omega_{i}\right)$ is the tensorial Fresnel factor (Appendix A), and $\chi^{(2)}$ denotes the surface nonlinear susceptibility tensor. If the infrared frequency $\omega_{2}$ is tuned across the surface vibrational resonances, resonant enhancement of $\chi_{i j k}^{(2)}$ is expected, as seen from the expression

$$
\chi_{i j k}^{(2)}\left(\omega_{2}\right)=\chi_{\mathrm{NR}, i j k}^{(2)}+\sum_{q} \frac{A_{q, i j k}}{\omega_{2}-\omega_{q}+i \Gamma_{q}},
$$

where $\chi_{\mathrm{NR}, i j k}^{(2)}$ is a non-resonant background, $A_{q, i j k}, \omega_{q}$, and $\Gamma_{q}$ are the amplitude, resonant frequency, and damping constant, respectively, of the qth vibrational mode. In this work, we shall focus on the $\mathrm{OH}$ stretch vibrational modes.

The surface nonlinear susceptibility $\chi^{(2)}$ is related to the hyperpolarizability $\boldsymbol{\alpha}^{(2)}$ of individual molecules in the surface layer by

$$
\begin{aligned}
\chi^{(2)} & =N_{\mathrm{S}}\left\langle\boldsymbol{\alpha}^{(2)}\right\rangle \text { or } \\
\chi_{i j k}^{(2)} & =N_{\mathrm{S}} \sum_{l m n} \alpha_{l m n}\langle(\hat{i} \cdot \hat{l})(\hat{j} \cdot \hat{m})(\hat{k} \cdot \hat{n})\rangle,
\end{aligned}
$$


where $N_{\mathrm{S}}$ is the surface density of molecules, the subindices $l, m, n$ refer to the molecular reference frame, and the angular brackets denote an orientational average over molecules. Since $\boldsymbol{\alpha}^{(2)}$ is expected to have the same resonent behavior as $\chi^{(2)}$, we have

$$
\alpha_{l m n}^{(2)}\left(\omega_{2}\right)=\alpha_{\mathrm{NR}, l m n}^{(2)}+\sum_{q} \frac{a_{q, l m n}}{\omega_{2}-\omega_{q}+i \Gamma_{q}},
$$

and

$$
A_{q, i j k}=N_{\mathrm{S}} \sum_{l m n} a_{q, l m n}\langle(\hat{i} \cdot \hat{l})(\hat{j} \cdot \hat{m})(\hat{k} \cdot \hat{n})\rangle .
$$

In the rapid-motion limit [38], however, we can show that $A_{q, i j k}$ becomes

$$
A_{q, i j k}=N_{\mathrm{S}} \sum_{l m n} a_{q, l m n}\langle(\hat{i} \cdot \hat{l})(\hat{j} \cdot \hat{m})\rangle\langle(\hat{k} \cdot \hat{n})\rangle,
$$

as we shall discuss in a later section. Note that in Eqs. (2.5) and (2.6) we have neglected the microscopic local field correction which, when fully included, will lead to the relacement of $N_{\mathrm{S}}$ in Eqs. (2.5) and (2.6) with $N_{\mathrm{S}} l_{\|}\left(\omega_{s}\right) l_{\|}\left(\omega_{1}\right) l_{\|}\left(\omega_{2}\right)$. The definition of the local-field correction factor $l_{\|}\left(\omega_{i}\right)$ is given in Appendix A.

The underlying idea of using SFG vibrational spectroscopy to study the ice surface structure is straightforward. Fig. 1(a) shows the structure of an ideal (0001) basal surface of ice $I_{h}$, which is likely to be the case at low temperatures when surface melting does not occur. In contrast, Fig. 1(b) shows a molecular dynamics simulation of the ice (0001) surface [13] with a quasi-liquid layer. We expect that the orientational distribution of the water molecules at the surface is very narrow if the surface layer has an ordered crystalline structure as in Fig. 1(a) but would broaden appreciably if the layer becomes liquid-like as in Fig. 1(b). Since the surface density of ice $N_{\mathrm{S}}$ is known, the microscopic amplitude $a_{q, l m n}$ for free $\mathrm{OH}$ stretch mode can be calculated (Sec. IIB), and the macroscopic amplitude $A_{q, i j k}$ can be obtained from experiment, we can deduce an approximate orientational distribution of the free $\mathrm{OH}$ bonds through Eq. (2.5) or (2.6). This then allows determination of the temperature variation in the orientational distribution of the molecules at the ice surface. 


\section{B. Molecular hyperpolarizability of $\mathrm{OH}$ bonds}

Since our quantitative analysis later will be on the free $\mathrm{OH}$ stretch mode, we present here our theoretical model of the molecular hyperpolarizability of a free $\mathrm{OH}$ bond. The theory of SFG vibrational spectroscopy shows that $a_{q, l m n}$ is related to the infrared and Raman characteristics of a vibrational mode through the equation [39]

$$
a_{q, l m n}=-\frac{1}{2 \epsilon_{0} \omega_{q}} \frac{\partial \mu_{n}}{\partial Q_{q}} \frac{\partial \alpha_{l m}^{(1)}}{\partial Q_{q}},
$$

where $\frac{\partial \mu_{n}}{\partial Q_{q}}$ and $\frac{\partial \alpha_{l m}^{(1)}}{\partial Q_{q}}$ are the infrared dipole derivative and the Raman polarizability tensor of the $q$ th vibrational mode, and $Q_{q}$ is the normal coordinate. For a free $\mathrm{OH}$ bond,

$$
Q_{q}=\sqrt{m_{\mathrm{H}}} \Delta r
$$

where $\Delta r$ is the change in the $\mathrm{OH}$ bond length due to stretch from equilibrium, and $m_{\mathrm{H}}$ is the mass of a hydrogen atom. Equation (2.7) then becomes

$$
a_{q, l m n}=-\frac{1}{2 \epsilon_{0} \omega_{q} m_{\mathrm{H}}} \frac{\partial \mu_{n}}{\partial \Delta r} \frac{\partial \alpha_{l m}^{(1)}}{\partial \Delta r}
$$

We can use Eq. (2.9) to calculate $a_{q, l m n}$ for the free $\mathrm{OH}$ stretch mode. The inset of Fig. 2 shows the molecular reference frame of a single $\mathrm{OH}$ bond. The infrared dipole derivative along the bond direction $\hat{\zeta}$ was reported to be

$$
\frac{\partial \mu_{\zeta}}{\partial \Delta r}=0.157(\text { atomic units })=2.52 \times 10^{-20} \mathrm{C}
$$

from an ab initio calculation [40], in good agreement with experiment [41]. For the Raman polarizability tensor, we use the experimentally deduced values from Ref. [42] (converted to MKS units)

$$
\begin{array}{r}
\frac{\partial \alpha_{\zeta \zeta}^{(1)}}{\partial \Delta r}=(2.20 \pm 0.13) \times 10^{-30} \frac{\mathrm{mC}}{\mathrm{V}} \\
\frac{\partial \alpha_{\xi \xi}^{(1)}}{\partial \Delta r}=\frac{\partial \alpha_{\eta \eta}^{(1)}}{\partial \Delta r}=(7.0 \pm 0.4) \times 10^{-31} \frac{\mathrm{mC}}{\mathrm{V}}
\end{array}
$$

with the depolarization ratio $r=\frac{\partial \alpha_{\xi \xi}^{(1)}}{\partial \Delta r} / \frac{\partial \alpha_{\zeta \zeta}^{(1)}}{\partial \Delta r} \approx 0.32$. The free $\mathrm{OH}$ stretch is at $\sim 3700 \mathrm{~cm}^{-1}$ [32], which corresponds to an angular frequency 


$$
\omega_{q}=2 \pi c \times 3700 \mathrm{~cm}^{-1}=7.0 \times 10^{14} \mathrm{sec}^{-1} .
$$

Inserting these values into Eq. (2.9), we obtain

$$
\begin{aligned}
a_{q, \zeta \zeta \zeta} & \approx 2.7 \times 10^{-27} \frac{\mathrm{m}^{4}}{\mathrm{Vsec}}, \\
a_{q, \xi \xi \zeta}=a_{q, \eta \eta \zeta}=r a_{q, \zeta \zeta \zeta} & \approx 8.6 \times 10^{-28} \frac{\mathrm{m}^{4}}{\mathrm{Vsec}} .
\end{aligned}
$$

\section{Symmetry of the ice basal surface}

Being a rank-3 tensor, $\chi_{i j k}^{(2)}$ (or $A_{q, i j k}$ ) has in general 27 elements. Surface symmetry, however, can make some elements vanish and some mutually dependent. The hexagonal symmetry of the (0001) surface of ice $I_{h}$ reduces the nonvanishing independent tensor elements to only three (see Fig. 2 for the reference frame):

$$
\begin{aligned}
& \chi_{z z z}^{(2)}, \\
& \chi_{x x z}^{(2)}=\chi_{y y z}^{(2)}, \\
& \chi_{x z x}^{(2)}=\chi_{z x x}^{(2)}=\chi_{y z y}^{(2)}=\chi_{z y y}^{(2)} .
\end{aligned}
$$

In the last equation, we have used the approximation $\chi_{i j k}^{(2)}=\chi_{j i k}^{(2)}$, assuming that the visible $\omega_{1}$ and the sum-frequency $\omega_{s}$ are far away from electronic resonances. In this case, the symmetry is the same as that for an azimuthally isotropic surface; in other words, SFG measurement cannot distinguish hexagonal and isotropic surface symmetries. The three independent components of $\chi_{i j k}^{(2)}$ can be determined from measurements with three input/output polarization combinations: $s s p$ (denoting $s-, s^{-}$, and $p$-polarized sum-frequency output, visible input, and infrared input, respectively), ppp, and sps through the relations derived from Eqs. (2.1) and (2.2)

$$
\begin{aligned}
& A_{q, \mathrm{eff}}(s s p)=\sin \beta_{2} L_{y y}\left(\omega_{s}\right) L_{y y}\left(\omega_{1}\right) L_{z z}\left(\omega_{2}\right) A_{q, x x z}, \\
& A_{q, \mathrm{eff}}(s p s)=\sin \beta_{1} L_{y y}\left(\omega_{s}\right) L_{z z}\left(\omega_{1}\right) L_{y y}\left(\omega_{2}\right) A_{q, x z x},
\end{aligned}
$$




$$
\begin{aligned}
& A_{q, \mathrm{eff}}(p p p) \\
& \quad=\sin \beta_{s} \sin \beta_{1} \sin \beta_{2} L_{z z}\left(\omega_{s}\right) L_{z z}\left(\omega_{1}\right) L_{z z}\left(\omega_{2}\right) A_{q, z z z} \\
& \quad-\cos \beta_{s} \cos \beta_{1} \sin \beta_{2} L_{x x}\left(\omega_{s}\right) L_{x x}\left(\omega_{1}\right) L_{z z}\left(\omega_{2}\right) A_{q, x x z} \\
& \quad+\sin \beta_{s} \cos \beta_{1} \cos \beta_{2} L_{z z}\left(\omega_{s}\right) L_{x x}\left(\omega_{1}\right) L_{x x}\left(\omega_{2}\right) A_{q, x z x} \\
& \quad-\cos \beta_{s} \sin \beta_{1} \cos \beta_{2} L_{x x}\left(\omega_{s}\right) L_{z z}\left(\omega_{1}\right) L_{x x}\left(\omega_{2}\right) A_{q, x z x},
\end{aligned}
$$

where $\beta_{s}, \beta_{1}$, and $\beta_{2}$ are the incidence angles of sum-frequency output, visible input, and infrared input, respectively.

In Ref. [43] Geiger and co-workers stated that the (0001) surface of ice is analogous to the $\operatorname{Si}(111)$ surface with $3 m\left(C_{3 \mathrm{v}}\right)$ symmetry [44] and possesses an additional independent nonvanishing element $\chi_{x x x}^{(2)}=-\chi_{x y y}^{(2)}=-\chi_{y x y}^{(2)}=\chi_{y y x}^{(2)}$. We note, however, that there is an essential difference between the two surfaces. As shown in Fig. 1(a), for an ideal (0001) surface of ice, the two adjacent layers A and layer B do not repeat each other by a simple spatial translation, but are rotated by $180^{\circ}$ with respect to each other. This is not the case for the $\operatorname{Si}(111)$ surface, where adjacent layers always repeat each other by a simple spatial translation. Due to its dynamic nature, the vapor/ice (0001) interface cannot be atomically flat, but contains steps. The two configurations A and B are energetically equivalent, and therefore, domains of type A and type B coexist with equal coverage. Although each type exhibits $3 m\left(C_{3 \mathrm{v}}\right)$ symmetry, the overall ice (0001) surface has a 6-fold symmetry instead of 3-fold, in strong contrast to the $\operatorname{Si}(111)$ surface.

\section{EXPERIMENT}

Ice $I_{h}$ single crystals were grown by the Bridgeman method from deionized water. The crystal growth apparatus was similar to the one described in Ref. [45]. Typical growth rate was $1 \mu \mathrm{m} / \mathrm{sec}(3.6 \mathrm{~mm} /$ hour $)$ to prevent bubbles. The ice crystals were then stored in a cold glove box and the storage temperature was between -5 and $-10{ }^{\circ} \mathrm{C}$. The sample preparation 
was then carried out in this glove box. The orientation of an ice crystal could be determined by locating the optical axis of the crystal using crossed polarizers.

To prepare the (0001) basal surface, an ice crystal was first cut (by melting) and roughly oriented with its (0001) surface exposed. It was then covered with a glass beaker containing pure ice and cooled slightly with a peltier cooler to let the crystal grow slowly from the vapor. After typically one day of growth, hexagonal facets appeared on the crystal surface. This allowed an accurate determination of the crystal orientation.

After the ice crystal was cut and oriented to have the (0001) surface (with a miscut less than $2^{\circ}$ ), it was "glued" (by melting and refreezing) to a clean fused silica substrate. Another fused silica plate, which had been coated with an octadecyltrichlorosilane (OTS) monolayer [46], was heated and brought in contact with the (0001) surface on the other side of the ice crystal. A bubble-free fused silica/OTS/ice interface was formed by melting and regrowing a thin layer of ice crystal at the interface. Finally the sample was put in a sealed cold chamber (Fig. 3) filled with ambient pressure air and brought to the SFG setup. The ice chamber was made of copper and could be cooled to $173 \mathrm{~K}$ with an accuracy of $0.1 \mathrm{~K}$. After cooling and waiting for the ice sample to reach thermal equilibrium with the chamber, the OTS-coated fused silica plate was separated from the ice sample by a mechanical device inside the chamber, leaving a smooth (0001) ice surface in equilibrium with the saturated water vapor in the sealed chamber. Such an ice surface preparation procedure is similar to that described in the glancing angle X-ray diffraction experiment [23] except that we used an OTS monolayer on the fused silica plate to make it hydrophobic and therefore much easier to separate from the ice crystal.

Since air was present in the ice chamber, the vapor/ice (or vapor/water) interface that we studied could be called an air/ice (or air/water) interface as well. Here we will not distinguish the subtle difference. Besides the vapor/ice interface, we also studied the interfaces between ice and fused silica plates which were either coated with OTS (hydrophobic) or uncoated (hydrophilic). These two interfaces will be called the silica/OTS/ice interface (Sec. V A) and the silica/ice interface (Sec. V B), respectively. 
The SFG experimental setup has been described elsewhere [31]. In this experiment, a $1.5 \mathrm{~mJ}$ visible laser pulse with a wavelength of $532 \mathrm{~nm}$ and a $100 \mu \mathrm{J}$ infrared pulse tunable from $2900 \mathrm{~cm}^{-1}$ to $3850 \mathrm{~cm}^{-1}$ (with a linewidth $\approx 6 \mathrm{~cm}^{-1}$ ) were overlapped at the sample surface with incidence angles of $45^{\circ}$ and $57^{\circ}$, respectively (Fig. 2). Both input pulses had a beam diameter of $\sim 1 \mathrm{~mm}$, a pulse duration of $\sim 15$ ps and a repetition rate of $20 \mathrm{~Hz}$. The SFG output was detected in the reflected direction. Precaution was taken in the experiment to avoid laser heating and damage. In the strong infrared absorption range of ice $\left(2900 \mathrm{~cm}^{-1}\right.$ to $\left.3570 \mathrm{~cm}^{-1}\right)$, the infrared input pulse energy was reduced to about $20 \mu \mathrm{J}$ to avoid laser heating. Furthermore, the sample chamber was put on a computer-controlled two-dimensional raster stage to prevent input pulses from repeatedly hitting the same spot. With such precautions, the SFG output was found to be linearly proportional to the infrared input energy, indicating negligible laser heating or damage (see Sec. VI A for more discussion on the effect of laser heating).

\section{STUDY OF THE VAPOR(AIR)/ICE INTERFACE}

\section{A. Experimental results}

Shown in Fig. 4 is a typical SFG spectrum of the vapor/ice (0001) interface at $232 \mathrm{~K}$ in comparison with that of the liquid water surface at $293 \mathrm{~K}$ (an enlarged water spectrum can be found in Ref. [38]). The polarization combination is ssp. The SFG output was calibrated against a reference $z$-cut quartz crystal [37], and corrected by the reflection loss at the windows on the ice chamber, yielding the spectra of $\left|\chi_{\mathrm{eff}}^{(2)}\right|^{2}$ in MKS units. As seen in Fig. 4, the spectrum of the ice surface is dominated by a strong but relatively broad peak at $\sim 3150 \mathrm{~cm}^{-1}$ that resembles the main $\mathrm{OH}$ stretch peak observed in the Raman spectrum of ice $\mathrm{I}_{\mathrm{h}}[47]$. This peak is associated with the bonded $\mathrm{OH}$ stretching modes in the

hydrogen bonding lattice. Unlike linear vibrational spectroscopy, in which the area under a resonant peak closely corresponds to the number of contributing oscillators $N$, in an SFG 
spectrum with negligible nonresonant background, the intensity or area of a resonant peak is proportional to $N^{2}$ and very sensitive to the degree of polar order. This explains why the intensity of the bonded $\mathrm{OH}$ peak from the vapor/ice interface appears to be more than 30 times higher than that from the vapor/water interface, which is not the case in the infrared absorption and Raman spectra of ice and water. Besides the bonded OH peak, the SFG spectra in Fig. 4 also exhibit a sharp peak around $3695 \mathrm{~cm}^{-1}$ associated with the stretch vibration of the free $\mathrm{OH}$ (or dangling $\mathrm{OH}$ ) bonds protruding from the surfaces [32].

Figures 5, 6 and 7 display the temperature dependence of the SFG spectra of the vapor/ice (0001) interface with polarization combinations ssp, ppp, and sps, respectively. In Fig. 5, the bonded $\mathrm{OH}$ peak decreases as the temperature increases but remains significantly different from that of liquid water (shown in the inset of Fig 4). It also shows a small frequency shift as the temperature increases, which is consistent with the temperature dependence of the bonded $\mathrm{OH}$ stretch frequency measured by Raman spectroscopy [48]. At this point, we do not know how the temperature dependence of the bonded $\mathrm{OH}$ peak in SFG is related to the appearance and change of the quasi-liquid layer on the ice surface. A molecular dynamics calculation is probably needed to find the answer.

The free $\mathrm{OH}$ peak in the SFG spectra is associated with the free $\mathrm{OH}$ bonds that can only exist at the ice surface or interface. Therefore, it can be used to characterize the surface or interface. Figure 8 displays a set of $s s p$ and $p p p$ spectra of the free $\mathrm{OH}$ peak for the vapor/ice interface at different temperatures. It is seen that the $p p p$ spectrum exhibits a strong temperature variation. The solid lines Fig. 8 are fits to the spectra using

$$
\chi_{\mathrm{eff}}^{(2)}\left(\omega_{2}\right)=\chi_{\mathrm{NR}, \mathrm{eff}}^{(2)}+\sum_{q} \frac{A_{q, \mathrm{eff}}}{\omega_{2}-\omega_{q}+i \Gamma_{q}} .
$$

The fitting parameters $A_{q, \text { eff }}(s s p)$ and $A_{q, \text { eff }}(p p p)$ are plotted in Fig. $9($ a) as functions of temperature (including data points deduced from spectra measured at many temperatures not shown in Fig. 8). Note that fitting the spectra with Eq. (2.2) could yield $\left|A_{q \text {,eff }}\right|$ but not the sign. The relative sign of $A_{q, \text { eff }}(s s p)$ and $A_{q \text {,eff }}(p p p)$ was obtained from an interference measurement between $A_{q, \text { eff }}(s s p)$ and $A_{q, \text { eff }}(p p p)$ using mixed polarizations for the visible 
input and SFG output. $A_{q, \text { eff }}(s s p)$ and $A_{q, \text { eff }}(p p p)$ were found to have the same sign.

In Fig. 9(a), $A_{q, \text { eff }}(s s p)$ and $A_{q \text {,eff }}(p p p)$ display relatively large fluctuations. This is because the experiment lasted for many days during which the ambient conditions were not controlled well. For example, the humidity in the room might have varied resulting in a relatively large uncertainty in the absolute SFG peak due to the water vapor absorption in the infrared beam path (see Fig. 10). However, these conditions affect $A_{q, \text { eff }}(s s p)$ and $A_{q, \text { eff }}(p p p)$ in a similar way and, therefore, have less effect on the ratio of the two. It can be seen in Fig. 9(a) that the fluctuations of $A_{q, \text { eff }}(s s p)$ and $A_{q, \text { eff }}(p p p)$ are correlated, with the consequence of smaller fluctuations in their ratio.

As we shall see in the next section, from the values of $A_{q, \text { eff }}(s s p)$ and $A_{q, \text { eff }}(p p p)$ and their ratio in Fig. 9(a) we can deduce an approximate orientational distribution for the free

$\mathrm{OH}$ bonds. The ratio $A_{q, \text { eff }}(p p p) / A_{q, \text { eff }}(s s p)$ remains constant at temperatures below $200 \mathrm{~K}$ and decreases continuously as the temperature increases. This indicates that the vapor/ice interface undergoes a continuous structural change which starts around $200 \mathrm{~K}$.

\section{B. Analysis of the free $\mathrm{OH}$ bonds}

To proceed with a more quantitative analysis, we must first verify our theoretical speculations regarding the symmetry of the (0001) surface of ice $I_{h}$. If the surface indeed has a 6-fold symmetry, from our theory in Sec. II C, we do not expect to observe any SFG signal with polarization combinations $s s s, p p s, s p p$ and $p s p$. The SFG spectra of the vapor/ice interface with these forbidden polarization combinations are presented in Fig. 11, in comparison with the allowed polarization combinations $s s p, p p p$ and $s p s$. As predicted, no SFG signal with the forbidden polarization combinations was observed.

We must also determine whether the slow-motion limit [Eq. (2.5)] or the rapid-motion limit [Eq. (2.6)] better describes the dynamic nature of the vapor/ice interface. In our recent work [38], we have shown that the strong evidence of motional averaging effect on the free $\mathrm{OH}$ stretch mode is in the unexpectedly weak SFG signal with the sps polarization 
combination. Figure 7 shows the sps SFG spectra of the vapor/ice interface, in which the resonant signal of the free $\mathrm{OH}$ stretch is hardly detectable at all temperatures. This resembles the case of the vapor/water interface [38] and indicates that the rapid-motion limit is a better approximation for describing the orientational fluctuations of the free $\mathrm{OH}$ bonds at the vapor/ice interface. From Eq. (2.6) with the rapid-motion approximation, we find

$$
\begin{aligned}
& A_{q, x x z}=A_{q, y y z} \\
& =\frac{1}{2} N_{\mathrm{S}}\left[a_{\zeta \zeta \zeta}\left(1-\left\langle\cos ^{2} \theta\right\rangle\right)+a_{\xi \xi \zeta}\left(1+\left\langle\cos ^{2} \theta\right\rangle\right)\right]\langle\cos \theta\rangle, \\
& A_{q, z z z}=N_{\mathrm{S}}\left[a_{\zeta \zeta \zeta}\left\langle\cos ^{2} \theta\right\rangle+a_{\xi \xi \zeta}\left(1-\left\langle\cos ^{2} \theta\right\rangle\right)\right]\langle\cos \theta\rangle, \\
& A_{q, x z x}=A_{q, y z y}=0 .
\end{aligned}
$$

Here $N_{\mathrm{S}}$ is the surface density of the free $\mathrm{OH}$ bonds; the angle brackets represent the ensemble average over the polar orientational distribution, $f(\theta)$, of the free $\mathrm{OH}$ bonds with the tilt angle $\theta(0 \leq \theta \leq \pi / 2)$ away from the surface normal (the azimuthal distribution is isotropic); and $a_{\zeta \zeta \zeta}$ and $a_{\xi \xi \zeta}=a_{\eta \eta \zeta}$ are the two independent nonvanishing hyperpolarizability elements of a free $\mathrm{OH}$ bond (values listed in Eq. (2.13)).

If we simply assume a truncated flat distribution for $f(\theta)$,

$$
\begin{array}{ll}
f(\theta)=\text { constant } & \text { for } 0 \leq \theta \leq \theta_{\mathrm{M}}, \\
f(\theta)=0 & \text { for } \theta>\theta_{\mathrm{M}},
\end{array}
$$

which yields

$$
\begin{aligned}
\langle\cos \theta\rangle & =\left(1+\cos \theta_{\mathrm{M}}\right) / 2, \\
\left\langle\cos ^{2} \theta\right\rangle & =\left(1+\cos \theta_{\mathrm{M}}+\cos ^{2} \theta_{\mathrm{M}}\right) / 3,
\end{aligned}
$$

then we can calculate $A_{q, x x z}$ and $A_{q, z z z}$ for each value of $\theta_{\mathrm{M}}$ using Eq. (4.2), from which we can further calculate $A_{q, \mathrm{eff}}(s s p)$ and $A_{q, \mathrm{eff}}(p p p)$ through Eq. (2.15). The values of the Fresnel 
factors used in this calculation are listed in Table I. The results of $A_{q, \text { eff }}(s s p)$ and $A_{q, \text { eff }}(p p p)$ from the calculation are presented in Fig. 9(a) as functions of $\theta_{\mathrm{M}}$. Note that we had some unknown (therefore adjustable) parameters in this calculation. One is the dielectric constant $\epsilon_{i}^{\prime}$ of the surface monolayer, as shown in Table I, and another is the factor $l_{\|}\left(\omega_{s}\right) l_{\|}\left(\omega_{1}\right) l_{\|}\left(\omega_{2}\right)$, which has been neglected in Eq. (2.6). The physical implications of $\epsilon_{i}^{\prime}$ and $l_{\|}\left(\omega_{i}\right)$ are discussed in Appendix A. In our calculation we assumed that $\epsilon_{s}^{\prime}=\epsilon_{1}^{\prime}$ (neglecting dispersion in the visible) and adjusted the value of $\epsilon_{1}^{\prime}$ such that the calculated ratio $A_{q \text {,eff }}(p p p) / A_{q, \text { eff }}(s s p)$ for $\theta_{\mathrm{M}}=0$ matches the experimentally deduced value $\sim 0.95$ at low temperatures (Fig. 9). At such low temperatures $(\leq 200 \mathrm{~K})$, the free $\mathrm{OH}$ bonds at the vapor/ice $(0001)$ interface presumably have a $\delta$-function-like orientational distribution perpendicular to the surface. From this we found $\epsilon_{s}^{\prime}=\epsilon_{1}^{\prime}=1.31$, which agrees very well with the estimate obtained from the model presented in the appendix of Ref. [49] [Eq. (A7) gives $\epsilon_{s}^{\prime}=\epsilon_{1}^{\prime}=1.29$ for ice]. The other parameters $\epsilon_{2}^{\prime}$ and $l_{\|}\left(\omega_{s}\right) l_{\|}\left(\omega_{1}\right) l_{\|}\left(\omega_{2}\right)$ cannot be determined experimentally, but fortunately, they do not sppear in the ratio $A_{q, \text { eff }}(p p p) / A_{q, \text { eff }}(s s p)$ although they still affact the absolute value of $A_{q \text {,eff }}$. For our estimation of the latter, we used the model in Ref. [49] and obtained $\epsilon_{2}^{\prime} \approx 1.18$ and $l_{\|}\left(\omega_{s}\right) l_{\|}\left(\omega_{1}\right) l_{\|}\left(\omega_{2}\right) \approx 1.32$.

With these estimated values of parameters, we could calculate the absolute value of $A_{q, \text { eff }}(s s p)$ for $\theta_{\mathrm{M}}=0$. From Eqs. (2.6) and (2.15) with the microscopic local-field correction included, we have

$$
\begin{aligned}
A_{q, \mathrm{eff}}(s s p)= & N_{\mathrm{S}} l_{\|}\left(\omega_{s}\right) l_{\|}\left(\omega_{1}\right) l_{\|}\left(\omega_{2}\right) \\
& \times \sin \beta_{2} L_{y y}\left(\omega_{s}\right) L_{y y}\left(\omega_{1}\right) L_{z z}\left(\omega_{2}\right) a_{q, \xi \xi \zeta} .
\end{aligned}
$$

The surface density of free $\mathrm{OH}$ bonds on the ice (0001) surface is one quarter of the surface density of $\mathrm{H}_{2} \mathrm{O}$ molecules because only the top half bilayer has broken hydrogen bonds and only half of the broken hydrogen bonds are dangling $\mathrm{OH}$ bonds (Fig. 1(a)). The surface density of $\mathrm{H}_{2} \mathrm{O}$ molecules in the top bilayer of the ice $(0001)$ surface is $1.14 \times 10^{15} \mathrm{~cm}^{-2}$, and one quarter of this value gives $N_{\mathrm{S}}=2.8 \times 10^{14} \mathrm{~cm}^{-2}$ (or 2.8/nmen $\mathrm{nm}^{2}$ Equation (4.5) then 
yields

$$
A_{q, \mathrm{eff}}(s s p)=1.29 \times 10^{-9} \mathrm{~m}^{2} \mathrm{~V}^{-1} \mathrm{sec}^{-1} .
$$

The measured $A_{q \text {,eff }}(s s p)$ at low temperatures is about $1.2 \times 10^{-9} \mathrm{~m}^{2} \mathrm{~V}^{-1} \mathrm{sec}^{-1}$ (Fig. 9). The agreement between theory and experiment here is remarkable considering the uncertainties in the input parameters we used in the calculation.

From Eqs. (2.15) and (4.2), the ratio of $A_{q, \text { eff }}(p p p) / A_{q \text {,eff }}(s s p)$ can also be calculated. The result as a function of $\theta_{\mathrm{M}}$ is given in Fig. 9(b). Comparing of the ratios $A_{q, \text { eff }}(p p p) / A_{q, \text { eff }}(s s p)$ obtained from the theory for different $\theta_{\mathrm{M}}$ (Fig. 9(b)) and from the experiment for different temperatures (Fig. 9(a)) yields $\theta_{\mathrm{M}}$ as a function of temperature, as shown in Fig. 12(a). Also shown in Fig. 12(a) is $\theta_{\mathrm{M}}$ of the free $\mathrm{OH}$ bonds at the vapor/water interface obtained in a similar way from the SFG spectra of the vapor/water interface (Fig. 13).

We can convert $\theta_{\mathrm{M}}$ into a more meaningful orientational order parameter $S$ defined as

$$
S=\frac{3\left\langle\cos ^{2} \theta\right\rangle-1}{2}=\frac{\cos \theta_{\mathrm{M}}+\cos ^{2} \theta_{\mathrm{M}}}{2}
$$

to describe the ordering of the free $\mathrm{OH}$ bonds. If $\theta_{\mathrm{M}} \rightarrow 0$, then $S \rightarrow 1$ corresponding to perfect orientational order. The other extreme is $\theta_{\mathrm{M}}=\pi / 2$ leading to $S=0$ for total disorder. One advantage of using $S$ instead of $\theta_{M}$ is that $S$ does not depend on the assumed distribution function $f(\theta)$. This can be seen from Eq. (4.2), which shows that the ratio $A_{q, z z z} / A_{q, y y z}$ depends only on $\left\langle\cos ^{2} \theta\right\rangle$ inrespective of the functional form of $f(\theta)$. The values of $S$ are displayed in Fig. 12(b). $S$ is close to 1 below $200 \mathrm{~K}$ and drops appreciably as the temperature increases above $200 \mathrm{~K}$.

\section{Implications for surface melting of ice}

An apparent onset of surface melting can be defined as the point at which surface disorder becomes detectable. Our experiment indicates that the onset of surface melting of ice is around $\sim 200 \mathrm{~K}$, below which the surface monolayer is still solid but above which it 
becomes increasingly disordered. Our onset temperature is lower than those obtained by other techniques. This is presumably because our SFG technique is more surface specific and sensitive to the disorder of the surface monolayer. For example, the recent measurement of x-ray scattering at glancing angles found that for the (0001) ice surface, the onset of surface melting was at $259.5 \mathrm{~K} \pm 2.5 \mathrm{~K}$, below which no surface melting could be observed [23]. However, the measurement did not have enough sensitivity to detect less than a few monolayers of quasi-liquid at the surface. Extrapolation of the data to zero quasi-liquid layer thickness to obtain the onset temperature for surface melting could yield too high a value.

The usual melting transition in the bulk is a first order phase transition, in which abrupt changes of physical properties are expected to occur. However, Fig. 12 shows that, within the experimental uncertainty, the decrease of the order parameter $S$ of the vapor/ice interface is continuous. This indicates that the quasi-liquid layer has a temperature-dependent structure and suggests that there should be a structural variation across the quasi-liquid layer on the ice surface. This picture is consistent with the results of molecular dynamics simulations $[12,14]$ but different from the simple models of surface melting used in the analyses of many experimental studies on ice. The latter assume a quasi-liquid layer with uniform structural properties.

An interesting feature displayed in Fig. 12 is that near the bulk melting temperature $(273 \mathrm{~K})$, the order parameter $S$ of the ice surface is even lower than that of the supercooled water surface. Although not physically impossible, this seems surprising and needs a satisfactory theoretical explanation. In any case, it indicates that the quasi-liquid layer on ice is different from the surface layer of water. There is a long-debated question whether surface melting of ice is complete or incomplete (i.e., whether the thickness of the quasi-liquid layer diverges or remains finite as the temperature approaches $273 \mathrm{~K}$ ) [19]. Our result here favors the incomplete surface melting scenario because otherwise one would expect the surface structure of the quasi-liquid layer to approach that of normal water as the layer thickness diverges. 


\section{STUDY OF OTHER ICE INTERFACES}

\section{A. Hydrophobic ice interface}

Presented in Fig. 14 is a set of SFG spectra for the fused-silica/OTS/ice (0001) interface at different temperatures. Since the interface is hydrophobic, the spectra appear similar to those for the vapor/ice interface, charaterized by a strong and broad hydrogen-bonded $\mathrm{OH}$ peak and a sharp free $\mathrm{OH}$ peak. An additional sharp peak at $\sim 2940 \mathrm{~cm}^{-1}$ is associated with a $\mathrm{CH}$ stretch mode of the methyl groups on OTS.

Figure 15 displays the SFG spectra for the free $\mathrm{OH}$ stretch in both $s s p$ and $p p p$ polarization combinations. Compared with the vapor/ice interface (Fig. 8), the free OH peak is shifted by $\sim 25 \mathrm{~cm}^{-1}$ to lower frequency due to the hydrophobic interaction with the OTS monolayer. Similar red shift of the free OD stretch at a hydrophobic surfactant $\mathrm{D}_{2} \mathrm{O}$ ice interface has been observed by infrared spectroscopy [50].

A major difference between the silica/OTS/ice and vapor/ice interfaces is that the free $\mathrm{OH}$ peak from the former remains very weak and hardly detectable for all temperatures with the $p p p$ polarization combination. Using the same analysis described earlier for the vapor/ice interface, we came to the conclusion that the dangling $\mathrm{OH}$ bonds at the silica/OTS/ice interface are highly disordered in orientation regardless of the temperature, presumably because the hydrophobic interaction with OTS on a fused fused (amorphous) silica plate forces the $\mathrm{OH}$ bonds to become orientationally disordered on a microscopic scale. When the silica plate was separated from the ice crystal, the exposed ice surface quickly relaxed to a more ordered structure that yielded a stronger free $\mathrm{OH}$ peak with the $p p p$ polarization combination (Fig. 8).

\section{B. Hydrophilic ice interface}

We also took the spectra of a hydrophilic ice interface by covering an ice surface with a clean (uncoated) fused silica plate. The silica plate was cleaned with acid, rinsed with 
deionized water and blown dry with nitrogen gas. Such a silica surface is known to be terminated by $\sim 5 / \mathrm{nm}^{2} \mathrm{SiOH}$ (silanol) groups, which can form hydrogen bonds with water and thus make the interface hydrophilic [51]. The $\mathrm{OH}$ stretch of the silanol groups on a hydrophilic silica surface is observable in the SFG spectrum [52], but is relatively weak.

The measured spectra of the silica/ice interface are presented in Fig. 16. The free $\mathrm{OH}$ peak has disappeared due to hydrogen bonding to the silanol groups on the silica surface, similar to the case of the silica/water interface [33]. The bonded $\mathrm{OH}$ peak dominated by contribution from ice, however, remains strong up to $272 \mathrm{~K}$. We also increased the sample temperature very slowly across the bulk melting temperature and obtained a thin layer of liquid water at the interface(spectrum denoted as $273^{+} \mathrm{K}$ in Fig. 16). An interesting transition was observed. As the interface started to melt, the bonded $\mathrm{OH}$ peak first broadened and decreased to a minimum, and then increased again. In the mean time another resonant peak around $3450 \mathrm{~cm}^{-1}$, characteristic of a liquid water surface, emerged.

A typical silica/water spectrum was reported earlier [33], in which the relatively strong SFG signal of the bonded $\mathrm{OH}$ stretch for water with low $p \mathrm{H}$ values was explained by polar ordering of the water molecules in the electric double layer created by the negative electric charge on the silica surface due to ionization of the silanol groups. Such an interpretation was verified by the change of the SFG spectrum with the $p H$ value of water. The negatively charged silica surface causes the majority of water molecules in the electric double layer to orient with their $\mathrm{OH}$ bonds (or dipole moments) towards the silica surface. In the case of the silica/ice interface, an electric double layer does not exist, and the strong bonded OH signal can only be explained by a net orientation of $\mathrm{OH}$ bonds pointing towards bulk ice, opposite to that in the electric double layer. Upon melting, the orientation of some water molecules at the interface flips, thus reducing the SFG signal. This explains the minimum intensity of the SFG signal in the melting transition. To confirm this interpretation, we performed a relative SFG phase measurement as illustrated in Fig. 17, based on the fact that the nonlinear susceptibility changes its sign when the average orientation of the molecules is inverted. This method was used previously to study molecular orientation of water molecules in the 
electric double layer at the silica/water interface as the $p \mathrm{H}$ value changes [53]. It relies on the interference pattern generated by interfering the SFG outputs from the sample and from a reference with the relative phase of the infrared inputs into the two varied by a modulator in the beam path. As the sample changed from silica/ice $(253 \mathrm{~K})$ into silica/water $\left(273^{+} \mathrm{K}\right)$, the interference pattern exhibited $\mathrm{a} \sim 180^{\circ}$ phase shift (Fig. 18), which confirmed that the hydrogen-bonded $\mathrm{OH}$ bonds at the silica/ice and silica/water interfaces are indeed oriented in opposite directions.

Interestingly, the strong bonded $\mathrm{OH}$ peak at the silica/ice interface can be greatly enhanced by doping the ice crystal with a small amount of ammonia, as shown in Fig. 19. The ammonia-doped ice crystal was grown from an ammonia-water solution with a $p \mathrm{H}$ value of 11.0 at room temperature. The crystal growth rate was $\sim 2 \mu \mathrm{m} / \mathrm{sec}$, twice as fast as the usual growth rate for growing pure ice crystals. Fast growth helps ammonia to incorporate into the ice crystal. A test sample was melted after growth to measure the actual doping concentration. The $p \mathrm{H}$ value of the melted test sample was 10.15 , corresponding to a $\mathrm{NH}_{3}$ doping concentration of $\sim 1 \mathrm{mM}$.

The enhancement of the bonded $\mathrm{OH}$ peak due to $\mathrm{NH}_{3}$ can be explained as follows. While the surface $\mathrm{NH}_{3}$ concentration could be significantly higher than that of the bulk, it was not high enough for the NH stretch peak to show up in the SFG spectrum (the NH symmetric stretch peak was reported to be at $3312 \mathrm{~cm}^{-1}$ in the SFG spectrum from the surface of ammonia-water solution [54]). However it could still have a dramatic effect on the net orientation of the $\mathrm{H}_{2} \mathrm{O}$ molecules near the silica/ice interface. As illustrated in Fig. 20, some $\mathrm{NH}_{3}$ molecules may form strong hydrogen bonding with the silanol groups on the silica surface with the nitrogen atoms facing silica, resulting in an excessive number of protons ( $\mathrm{NH}$ bonds) pointing into ice. Starting from these surface-anchored $\mathrm{NH}_{3}$ molecules, the neighoring $\mathrm{H}_{2} \mathrm{O}$ molecules in the ice lattice tend to adopt the polar orientations (with a net dipole moment pointing toward the bulk ice) to satisfy the Bernal-Fowler ice rules [55] until Bjerrum defects (broken hydrogen bonds with two protons facing each other) set in $[56,57]$. Consequently, net polar ordering of the $\mathrm{H}_{2} \mathrm{O}$ molecules is expected near the interface, which 
enhances the SFG signal. A similar picture was used to explain the extraordinarily strong bonded-OH peaks in the SFG spectrum from ice films grown on the $\operatorname{Pt}(111)$ surface [35].

\section{DISCUSSION}

\section{A. Laser heating}

One of the major concerns in our experiment was to ensure that the results shown in Fig. 12 are not perurbed by laser heating. Fortunately, ice does not absorb as strongly in the free $\mathrm{OH}$ stretch range as it does in the bonded $\mathrm{OH}$ range. The infrared penetration depth in ice is $\sim 40 \mu \mathrm{m}$ at $3700 \mathrm{~cm}^{-1}$, in comparison to less than $1 \mu \mathrm{m}$ at the peak absorption frequency $3250 \mathrm{~cm}^{-1}$. We tested the heating effect experimentally. Fig. 21 shows a set of SFG spectra of the vapor/ice interface with different infrared input energies. Attenuation of the infrared pulse was achieved by inserting silicon windows ( $2 \mathrm{~mm}$ thick, polished on both sides) in the infrared beam path and readjusting the time delay for beam overlapping. The effect of laser heating was found to be negligible in the free $\mathrm{OH}$ range, but in significant in the bonded $\mathrm{OH}$ range. By attenuating the infrared input to below $20 \%$ of its full power, laser heating became negligible even for ice at $172 \mathrm{~K}$. This was confirmed by the linear dependence of the SFG output on the input infrared energy. Therefore, we used in ourexpriments $20 \%$ of the full infrared power to obtain SFG spectra of ice in the bonded OH range $\left(2900 \mathrm{~cm}^{-1}\right.$ to $\left.3570 \mathrm{~cm}^{-1}\right)$.

\section{B. Surface roughening}

Roughening transition [58] that often results in the disappearance of facets and rounding up of the equilibrium crystal shape could also occur on a solid surface. Surface roughening is similar to surface melting in the sense that it causes disordering of a surface. To determine whether surface disordering observed by SFG is due to surface melting or surface roughening, we refer to other experiments. Elbaum studied surface roughening of ice with interference 
microscopy [59] and found that the roughening transition does not happen on the (0001) basal surface of ice at all temperatures up to the triple point, in contrast to the (1010) prism surface, on which a roughening transition was observed around $-2^{\circ} \mathrm{C}$. In our experiment, we used the (0001) surface and observed surface disordering at much lower temperatures than the temperature range reported in Ref. [59]. Surface roughening as the explanation can thus be ruled out.

We, however, observed another kind of roughening of the ice surface in our experiment, which is on a much larger length scale. We found that the smoothness of the vapor/ice interface appeared to deteriorate during the experiment, especially when the temperature was above $253 \mathrm{~K}$, due to extended exposure to the high intensity laser pulses. Although the effect of laser heating on the SFG spectra was confirmed to be negligible, mild laser heating was still present and could disturb the delicate dynamic equilibrium at the vapor/ice interface, resulting in a slow deterioration of the surface smoothness on a macroscopic scale. However, this kind of roughening reduced the free $\mathrm{OH}$ peak intensities in ssp and $p p p$ proportionally and therefore did not affect the ratio $A_{q \text {,eff }}(p p p) / A_{q \text {,eff }}(s s p)$, which is a measure of the surface order. This indicates that the SFG signal was still dominated by large (0001)

terraces at the vapor/ice interface. The reduction of the SFG signal due to such roughening was also approximately proportional to the reduction of specular reflection of the $532 \mathrm{~nm}$ visible input pulse. A photodiode was used in our experiment to monitor the smoothness of the vapor/ice interface by measuring the reflected visible pulse. The SFG signal could then be corrected by normalizing against the measured visible reflectivity when necessary, and the experiment terminated if the reflected visible reflectivity dropped below $2 / 3$ of that from a perfectly smooth ice surface.

\section{Effect of impurities}

It has been suggested that impurities may affect surface melting of ice $[11,19]$. In our study of the vapor/ice interface, however, the impurity effect due to contamination of the ice 
sample is unlikely to be significant. We note that in a recent experiment to demonstrate the effect of impurities, an ice sample containing a very high concentration (10 mM) of $\mathrm{KCl}$ was used [25]. Our ice crystals were grown and prepared under clean conditions, and therefore, concerns about ionic impurities with a high concentration seem to be irrelevant. It would be interesting to study the impurity effect by doping the ice crystals. With ammonia doping (Sec. VB), a concentration of $\sim 1 \mathrm{mM}$ of $\mathrm{NH}_{3}$ can be incorporated into the ice crystal. A much higher doping concentration (600 ppm or $30 \mathrm{mM}$ ) of $\mathrm{KOH}$ in ice has been reported (beyond which the ice crystal turns slightly milky) [60].

Some airborne impurities are more likely to be a source of contamination than ionic impurities since air was present in our experiment. One typical contaminant in atmosphere is the hydrocarbons. In our experience, this kind of impurity at the vapor/water or vapor/ice interface is likely to suppress the dangling $\mathrm{OH}$ bonds. In the current experiment, hydrocarbon contamination is unlikely because of the clear presence of the free $\mathrm{OH}$ bonds. Another air borne impurity is carbon dioxide $\left(\mathrm{CO}_{2}\right)$. We have tried to study the effect by intentionally dosing the ice chamber with $\mathrm{CO}_{2}$ gas. Unfortunately, $\mathrm{CO}_{2}$ has an infrared absorption band right at the free $\mathrm{OH}$ stretch frequency and prevented us from getting reliable SFG spectra from the $\mathrm{CO}_{2}$ /ice interface. This problem could be solved by reducing the infrared beam path in the ice chamber. Investigating the impurity effect on surface melting of ice is an ongoing project.

\section{CONCLUSION}

In summary, we have applied surface-specific $\mathrm{SFG}$ vibrational spectroscopy on ice $\left(\mathrm{I}_{\mathrm{h}}\right)$ interfaces with vapor, a hydrophilic silica substrate, and a hydrophobic OTS-surfactantcoated silica substrate. The results allow us to study surface melting of ice by probing the structure of monolayers at the (0001) surface of ice. Orientational disordering of water molecules at the vapor/ice interface as a signature of surface melting appears to set in

around $200 \mathrm{~K}$. The degree of disordering increases with temperature and indicates that the 
quasi-liquid layer on the ice surface is structurally different from a normal water surface layer. The SFG spectra in the free $\mathrm{OH}$ stretch mode have very different behaviors for the three different interfaces because of different interactions of the water molecules with the subsrates at the interfaces, but they have relatively similar temperature dependence in the bonded $\mathrm{OH}$ stretch range.

\section{ACKNOWLEDGMENTS}

This work was supported by the Director, Office of Energy Research, Office of Basic En-

ergy Sciences, Materials Sciences Division of the U.S. Department of Energy, under contract No. DE-AC03-76SF00098.

\section{APPENDIX A: TOTAL LOCAL-FIELD FACTORS AT THE SURFACE}

The $L_{i i}$ factors in Eq. (2.1) are the transmission Fresnel coefficients relating the field components in an input or reflected output beam in medium 1 to the corresponding ones in the interfacial layer between media 1 and 2 [31,61]. For an interface between two continuous media 1 and 2 with dielectric constants $\epsilon_{1}$ and $\epsilon_{2}$, we have

$$
\begin{aligned}
L_{X X}\left(\omega_{i}\right) & =\frac{2 \epsilon_{1}\left(\omega_{i}\right) k_{2 Z}\left(\omega_{i}\right)}{\epsilon_{2}\left(\omega_{i}\right) k_{1 Z}\left(\omega_{i}\right)+\epsilon_{1}\left(\omega_{i}\right) k_{2 Z}\left(\omega_{i}\right)}, \\
L_{Y Y}\left(\omega_{i}\right) & =\frac{2 k_{1 Z}\left(\omega_{i}\right)}{k_{1 Z}\left(\omega_{i}\right)+k_{2 Z}\left(\omega_{i}\right)}, \\
L_{Z Z}\left(\omega_{i}\right) & =\frac{2 \epsilon_{1}\left(\omega_{i}\right) \epsilon_{2}\left(\omega_{i}\right) k_{1 Z}\left(\omega_{i}\right)}{\epsilon_{2}\left(\omega_{i}\right) k_{1 Z}\left(\omega_{i}\right)+\epsilon_{1}\left(\omega_{i}\right) k_{2 Z}\left(\omega_{i}\right)} \cdot \frac{1}{\epsilon^{\prime}\left(\omega_{i}\right)},
\end{aligned}
$$

where $\epsilon^{\prime}\left(\omega_{i}\right)$ is an effective dielectric constant of the interfacial layer at $\omega_{i}$. From the theoretical point of view, the dielectric constant is not well defined for a monolayer because it is supposed to be a macroscopic or mesoscopic property. However, $\epsilon^{\prime}$ can be interpreted as a result of the microscopic local-field correction in the interfacial layer [49,62].

The microscopic local field components experienced by the interfacial layer are 


$$
\begin{aligned}
& E_{X}^{(\mathrm{Loc})}=l_{X X} L_{X X}^{\prime} E_{X}, \\
& E_{Y}^{(\mathrm{Loc})}=l_{Y Y} L_{Y Y}^{\prime} E_{Y}, \\
& E_{Z}^{(\mathrm{Loc})}=l_{Z Z} L_{Z Z}^{\prime} E_{Z},
\end{aligned}
$$

where $l_{i i}$ denotes the microscopic local-field correction factor, $L_{i i}^{\prime}$ is the Fresnel or macroscopic local-field factor (defined by Eq. (A1) but without the factor $\frac{1}{\epsilon^{\prime}}$ in $L_{Z Z}$ ), and $E_{i}$ is the electric field component of the incoming and outgoing optical plane waves in medium 1. If $l_{X X}=l_{Y Y}=l_{\|}$and $l_{Z Z}=l_{\perp}$, the total local-field factors including both macroscopic and microscopic effects are

$$
\begin{aligned}
& F_{X X}=l_{X X} L_{X X}=l_{\|} \frac{2 \epsilon_{1} k_{2 Z}}{\epsilon_{2} k_{1 Z}+\epsilon_{1} k_{2 Z}}, \\
& F_{Y Y}=l_{Y Y} L_{Y Y}=l_{\|} \frac{2 k_{1 Z}}{k_{1 Z}+k_{2 Z}}, \\
& F_{Z Z}=l_{Z Z} L_{Z Z}=l_{\perp} \frac{2 \epsilon_{1} \epsilon_{2} k_{1 Z}}{\epsilon_{2} k_{1 Z}+\epsilon_{1} k_{2 Z}} .
\end{aligned}
$$

We notice that $F_{i i}$ differs from $L_{i i}$ in Eq. (A1) only by a common factor $l_{\|}$if we define

$$
\epsilon^{\prime}=\frac{l_{\|}}{l_{\perp}}
$$

It has been shown that the value of $\epsilon^{\prime}$ defined this way is usually between 1 and the bulk dielectric constant $\epsilon$ [49]. The physical meaning of $\epsilon^{\prime}$ now becomes clear; it is simply the ratio of $l_{\|}$to $l_{\perp}$. By introducing the factor $\frac{1}{\epsilon^{\prime}}$ in Eq. (A1), we have partially included the microscopic local-field correction. To have it fully included, the surface density $N_{\mathrm{S}}$ in Eqs. (2.3), (2.5), (2.6) and (4.2) should all be replaced with $N_{\mathrm{S}} l_{\|}\left(\omega_{s}\right) l_{\|}\left(\omega_{1}\right) l_{\|}\left(\omega_{2}\right)$. The additional factor $l_{\|}\left(\omega_{s}\right) l_{\|}\left(\omega_{1}\right) l_{\|}\left(\omega_{2}\right)$ can be neglected if only the ratios of $A_{q, i j k}$ are used in the deduction of the orientational distribution function $f(\Omega)$.

A simple slab model was presented in the appendix of Ref. [49] to obtain an estimate of $\epsilon^{\prime}$ and $l_{\|}$. For the free surface of a medium with a bulk dielectric constant $\epsilon$, this slab model gives

$$
l_{\perp}=\frac{2 \epsilon+1}{3 \epsilon}
$$




$$
\begin{aligned}
l_{\|} & =\frac{\epsilon+5}{6}, \\
\epsilon^{\prime} & =\frac{\epsilon(\epsilon+5)}{4 \epsilon+2} .
\end{aligned}
$$




\section{TABLES}

TABLE I. Calculated Fresnel factors for the vapor/ice interface. $z$ is along the sample surface normal, with the $x z$ plane being the light incidence plane. $\beta$ is the incidence angle of the input or output beam. $\epsilon^{\prime}$ is the effective dielectric constant of the surface monolayer.

\begin{tabular}{l|ccc}
\hline \hline & $\omega_{s}$ & $\omega_{1}$ & $\omega_{2}$ \\
\hline$\lambda$ & $444 \mathrm{~nm}$ & $532 \mathrm{~nm}$ & $2.7 \mu \mathrm{m}$ \\
$n$ & 1.31 & 1.31 & 1.18 \\
$\beta$ & $46.8^{\circ}$ & $45^{\circ}$ & $57^{\circ}$ \\
\hline$L_{x x}$ & .96 & .95 & 1.04 \\
$L_{y y}$ & .77 & .78 & .79 \\
$L_{z z}$ & $1.04 / \epsilon_{s}^{\prime}$ & $1.05 / \epsilon_{1}^{\prime}$ & $.96 / \epsilon_{2}^{\prime}$ \\
\hline $\mathrm{e}_{x} L_{x x}(p)$ & -.66 & .67 & .57 \\
$\mathrm{e}_{y} L_{y y}(s)$ & .77 & .78 & .79 \\
$\mathrm{e}_{z} L_{z z}(p)$ & $.76 / \epsilon_{s}^{\prime}$ & $.74 / \epsilon_{1}^{\prime}$ & $.80 / \epsilon_{2}^{\prime}$ \\
\hline \hline
\end{tabular}




\section{REFERENCES}

* Present address: Lucent Technologies, Bell Labs 1D-410, 600 Mountain Ave., Murray Hill, NJ 07974.

† Present address: Departamento de Física, Universidade Estadual Paulista, C.P. 473, Bauru - SP 17015-970, Brazil.

[1] M. Faraday, Lecture before the Royal Institution reported in the Athenaeum no.1181, 640 (1850); M. Faraday, Philos. Mag. 17, 162 (1859); M. Faraday, Proc. Roy. Soc., London 10, 440 (1860).

[2] J. W. M. Frenken and J. F. van der Veen, Phys. Rev. Lett. 54, 134 (1985);

J. W. M. Frenken, P. M. J. Maree, and J. F. van der Veen, Phys. Rev. B 34, 7506 (1986).

[3] K. C. Prince, U. Breuer, and H. P. Bonzel, Phys. Rev. Lett. 60, 1146 (1988).

[4] D.-M. Zhu and J. G. Dash, Phys. Rev. Lett. 57, 2959 (1986).

[5] E. G. McRae and R. A. Malic, Phys. Rev. Lett. 58, 1437 (1987).

[6] R. Trittibach, Ch. Grütter, and J. H. Bilgram, Phys. Rev. B 50, 2529 (1994).

[7] M. Polčik, L. Wilde, and J. Haase, Phys. Rev. Lett. 78, 491 (1997).

[8] J. G. Dash, H. Fu, and J. S. Wettlaufer, Rep. Prog. Phys. 58, 115 (1995).

[9] J. S. Wettlaufer and J. G. Dash, Scientific American, 282, 34 (2000).

[10] N. H. Fletcher, Philos. Mag. 18, 1287 (1968).

[11] J. S. Wettlaufer, Phys. Rev. Lett. 82, 2516 (1999); and references therein.

[12] G.-J. Kroes, Surface Science 275, 365 (1992).

[13] Y. Furukawa and H. Nada, J. Phys. Chem. B 101, 6167 (1997). 
[14] K. Bolton and J. B. C. Pettersson, J. Phys. Chem. B 104, 1590 (2000).

[15] D. Nason and N. H. Fletcher, J. Chem. Phys. 62, 4444 (1975).

[16] I. Golecki and C. Jaccard, J. Phys. C 11, 4229 (1978).

[17] D. Beaglehole and D. Nason, Surf. Sci. 96, 357-65 (1980).

[18] Y. Furukawa, M. Yamamoto, and T. Kuroda, J. Cryst. Growth 82, 655 (1987).

[19] M. Elbaum, S. G. Lipson, and J. G. Dash, J. Cryst. Growth 129, 491-505 (1993).

[20] J. Ocampo and J. Klinger, J. Phys. Chem. 87, 4325 (1983)

[21] Y. Mizuno and N. Hanafusa, J. Phys. Colloq. C1 48, 511 (1987)

[22] N. Materer, U. Starke, A. Barbieri, M. A. Van Hove, G. A. Somorjai, G.-J. Kroes, and C. Minot, J. Phys. Chem. 99, 6267 (1995).

[23] H. Dosch, A. Lied, and J. H. Bilgram, Surf. Sci. 327, 145-164 (1995); A. Lied, H. Dosch, and J. H. Bilgram, Phys. Rev. Lett. 72, 3554 (1994).

[24] J. Braun, A. Glebov, A. P. Graham, A. Menzel, and J. P. Toennies, Phys. Rev. Lett. 80, 2638 (1998).

[25] A. Döppenschmidt and H. J. Butt, Langmuir 16, 6709 (2000).

[26] B. Pittinger, D. J. Cook, C. R. Slaughterbeck, and S. C. Fain, J. Vac. Sci. Technol. A 16, $1832(1998)$.

[27] R. R. Gilpin, J. Colloid Interface Sci. 77, 435 (1980).

[28] M. Maruyama, M. Bienfait, J. G. Dash, and G. Coddens, J. Cryst. Growth 118, 33 (1992).

[29] S. S. Barer, N. V. Churaev, B. V. Derjaguin, O. A. Kiseleva, and V. D. Sobolev, J. Colloid Interface Sci. 74, 173 (1980). 
[30] CRC Handbook of Chemistry and Physics, 62th edition (CRC Press, Roca Roton, FL, 1982), p.D-167.

[31] Y. R. Shen, "Surface spectroscopy by nonlinear optics", in Frontiers in Laser Spectroscopy, Proceedings of the International School of Physics "Enrico Fermi", Course CXX, T. W. Hänsch and M. Inguscio, eds., (North Holland, Amsterdam, 1994), p.139.

[32] Q. Du, R. Superfine, E. Freysz, and Y. R. Shen, Phys. Rev. Lett. 70, 2313 (1993).

[33] Q. Du, E. Freysz, and Y. R. Shen, Phys. Rev. Lett. 72, 238 (1994).

[34] Q. Du, E. Freysz, and Y. R. Shen, Science 264, 826 (1994).

[35] X. Su, L. Lianos, Y. R. Shen, and G. A. Somorjai, Phys. Rev. Lett. 80, 1533 (1998).

[36] X. Wei, P. B. Miranda, and Y. R. Shen, Phys. Rev. Lett., 86, 1554 (2001).

[37] X. Wei, S.-C. Hong, X. Zhuang, T. Goto, and Y. R. Shen, Phys. Rev. E 62, 5160 (2000).

[38] X. Wei and Y. R. Shen, Phys. Rev. Lett., 86, 4799 (2001).

[39] R. Superfine, J. Y. Huang, and Y. R. Shen, Chem. Phys. Lett. 172, 303 (1990).

[40] A. Morita and J. T. Hynes, Chem. Phys. 258, 371 (2000).

[41] S. A. Clough, Y. Beers, G. P. Klein, and L. S. Rothman, J. Chem. Phys. 59, 2254 (1973).

[42] W. F. Murphy, Mol. Phys. 36, 727 (1978).

[43] F. M. Geiger, A. C. Tridico, and J. M. Hicks, J. Phys. Chem. B 103, 8205 (1999).

[44] T. F. Heinz, M. M. T. Loy, and W. A. Thompson, Phys. Rev. Lett. 54, 63 (1985).

[45] R. A. Brown, J. Kelzer, U. Stelger, and Y. Yeh, J. Phys. Chem. 87, 4135 (1983)

[46] J. Sagiv, J. Am. Chem. Soc. 10292 (1980).

[47] J. R. Scherer and R. G. Snyder, J. Chem. Phys. 67, 4794 (1977). 
[48] P. T. T. Wong and E. Whalley, J. Chem. Phys. 62, 2418 (1975).

[49] X. Zhuang, P. B. Miranda, D. Kim, and Y. R. Shen, Phys. Rev. B59, 12632 (1999).

[50] I. Engquist and B. Liedberg, J. Phys. Chem. 100, 20089 (1996).

[51] R. K. Iler, The Chemistry of Silica (Wiley, New York, 1979) p.622-729.

[52] K. Wolfrum, J. Löbau, and A. Laubereau, Appl. Phys. A 59, 605 (1994).

[53] Y. R. Shen, Solid State Communications 108, 399 (1998).

[54] D. Simonelli and M. J. Shultz, J. Chem. Phys. 112, 6804 (2000).

[55] J. D. Bernal and R. H. Fowler, J. Chem. Phys. 1, 515 (1933).

[56] P. V. Hobbs, Ice Physics, (Oxford University Press, New York, 1974) Chap. 2.

[57] V. F. Petrenko and R. W. Whitworth, Physics of Ice, (Oxford University Press, New York, 1999) Chap. 4.

[58] M. C. Desjonquères and D. Spanjaard, Concepts in Surface Physics, 2nd edition (Springer, New York, 1996) p.15-43.

[59] M. Elbaum, Phys. Rev. Lett. 67, 2982 (1991).

[60] S. Kawada and R. Tutiya, J. Phys. Chem. Solids 58, 115 (1997).

[61] Y. R. Shen, Annu. Rev. Phys. Chem. 40, 327 (1989).

[62] P. Ye and Y. R. Shen, Phys. Rev. B 28, 4288 (1983). 

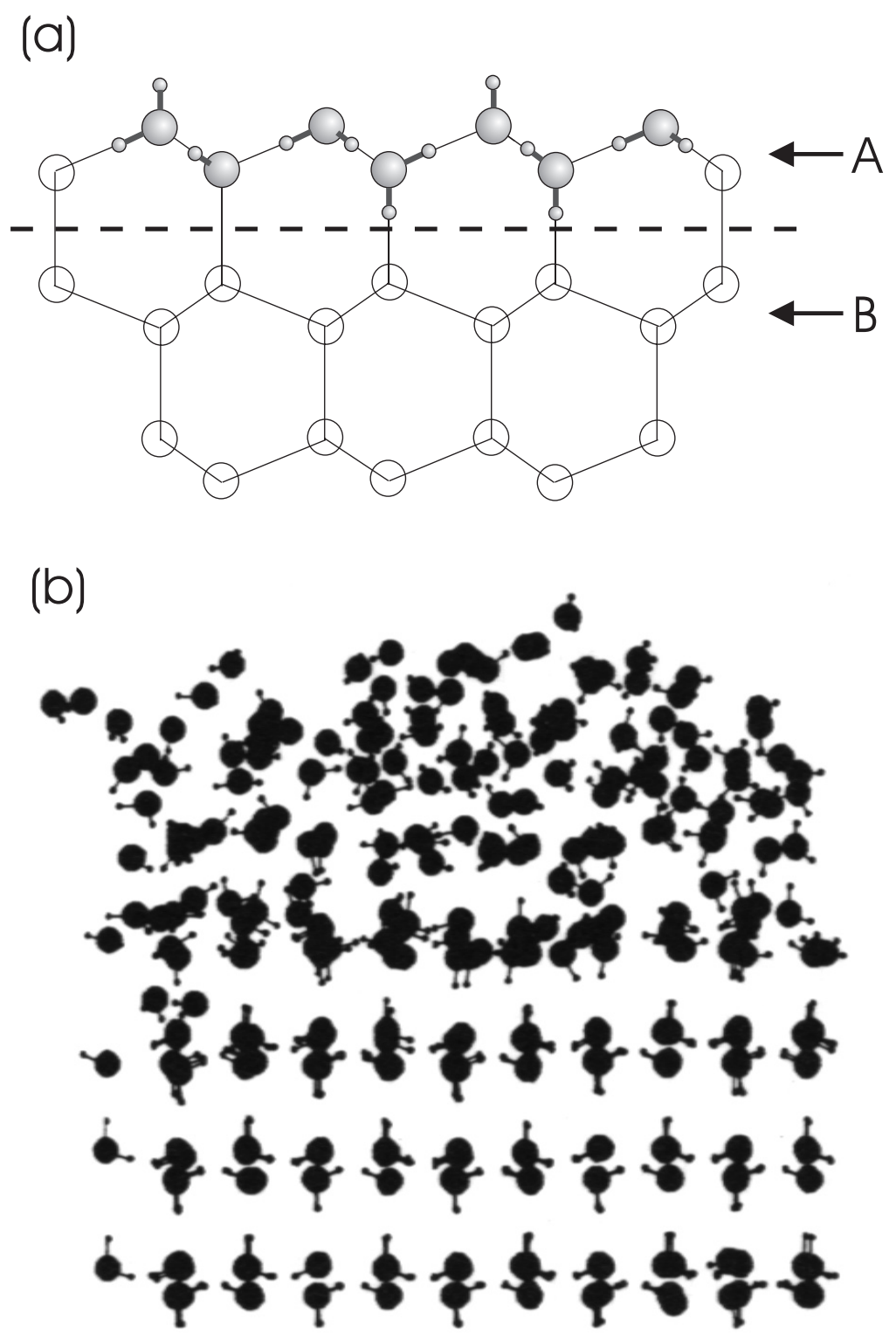

FIG. 1. (a) Side view of an ideal (0001) basal surface of ice $\mathrm{I}_{\mathrm{h}}$. Note that the free (or dangling) $\mathrm{OH}$ bonds are perpendicular to the surface due to the tetrahedral hydrogen bonding geometry. Also note that the adjacent layers A and B do not repeat each other by a simple spatial translation but are rotated by $180^{\circ}$ with respect to each other in the surface plane. (b) Molecular dynamics simulation of the (0001) basal surface of ice $\mathrm{I}_{\mathrm{h}}$ at $265 \mathrm{~K}$ (from Ref. [13]). 


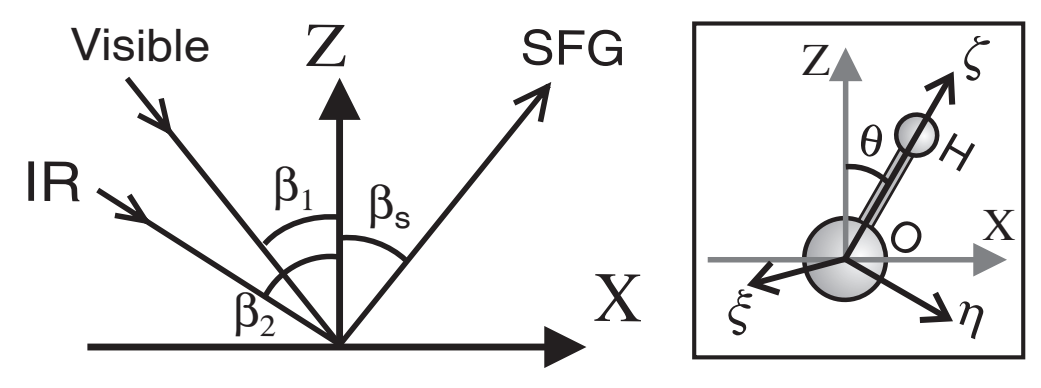

FIG. 2. Geometry of the input/output beams in the SFG experiment with $x, y, z$ describing the lab-frame coordinates. The right inset shows molecular reference frame of a single $\mathrm{OH}$ bond, with $\zeta$ along the bond direction and $\xi, \eta$ perpendicular to $\zeta$. The tilt angle $\theta$ is defined as the angle between $\zeta$ and the $z$-axis. 


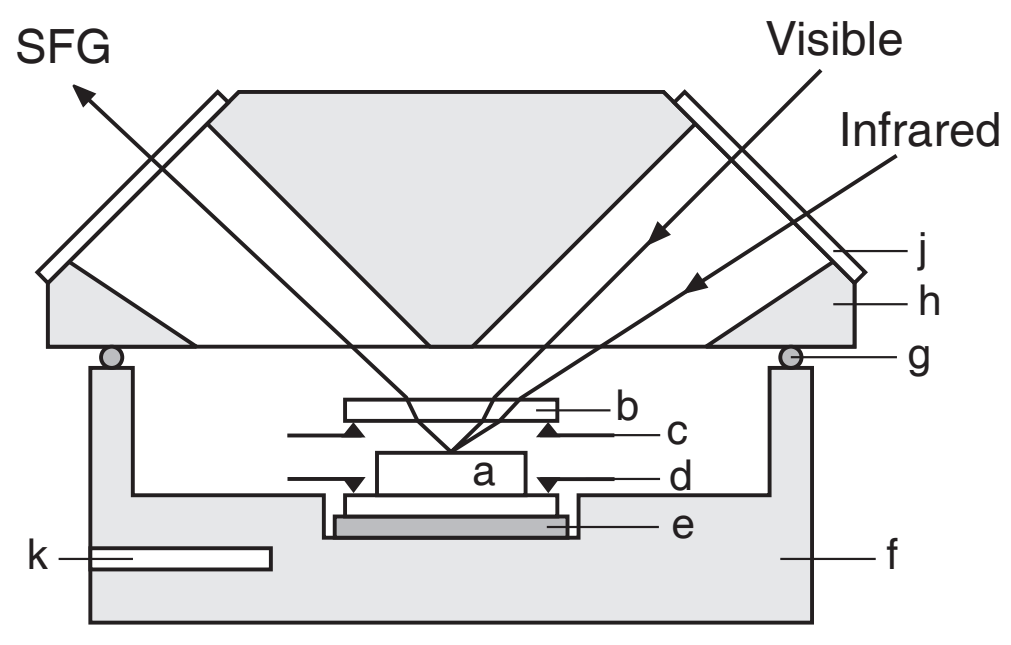

FIG. 3. Schematic drawing of the ice chamber. (a)Single-crystal ice sample frozen to a fused silica plate at the bottom. (b)OTS-coated fused silica plate that can be mechanically separated from the ice crystal. (c)Stainless steel levers that are used to lift the OTS-coated silica plate. (d)Stainless steel clamps that secure the bottom silica plate. (e)Glass color filter to absorb the transmitted visible input pulse. (f)Copper chamber cooled with cold nitrogen gas (cooling stage not shown). (g)O-ring. (h)Chamber cover made of Plexiglas. (j)Fused silica windows. (k)Omega Pt100 platinum resistance thermometer. 


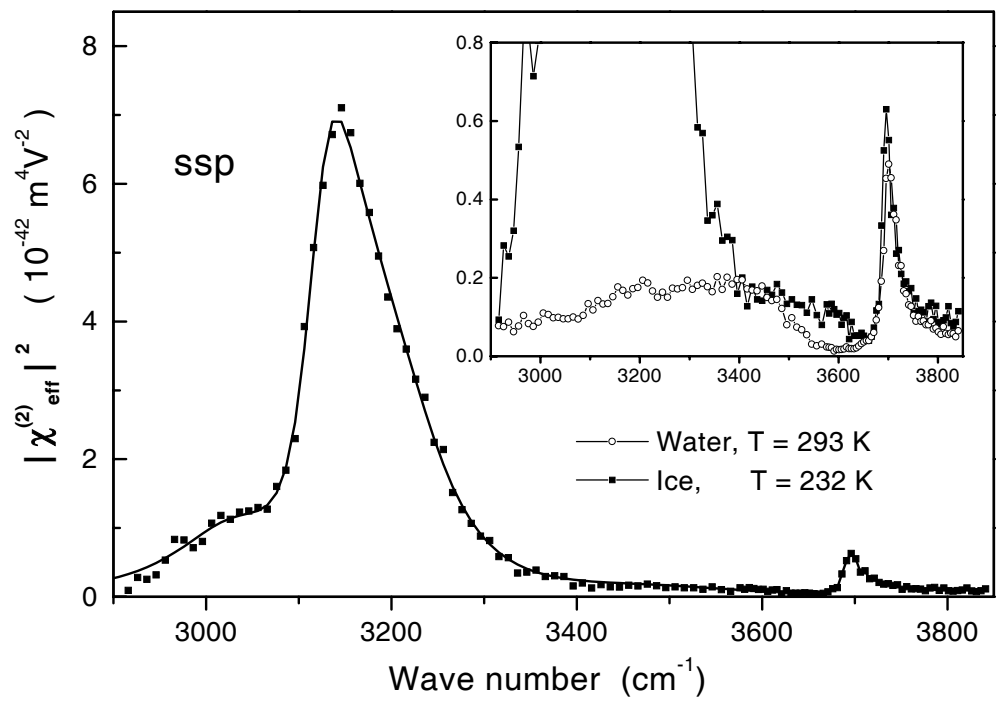

FIG. 4. SFG spectra of the vapor/ice (0001) interface at $232 \mathrm{~K}$ (squares) and the vapor/water interface at $293 \mathrm{~K}$ (circles). The polarization combination is $s s p$. 


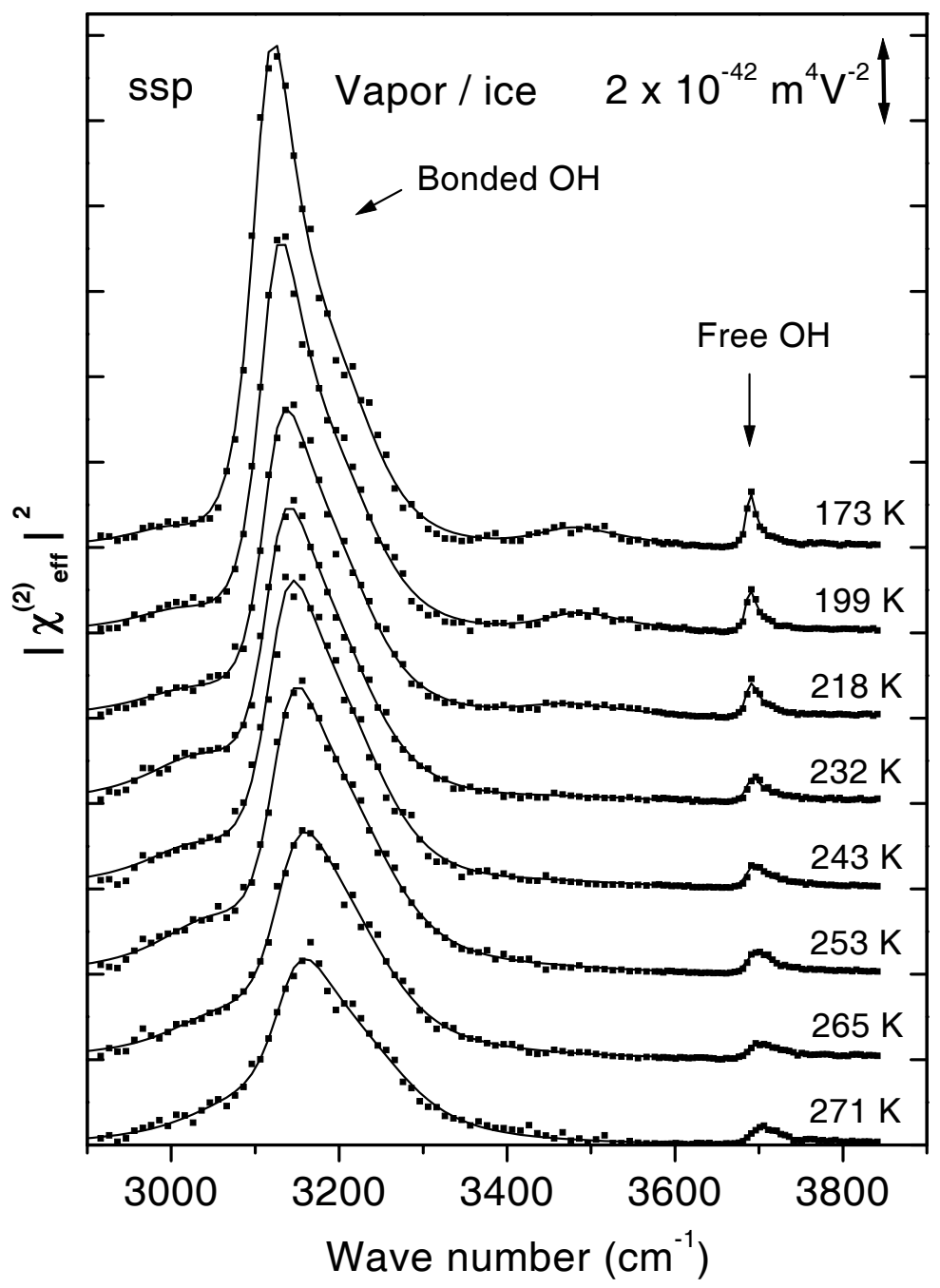

FIG. 5. SFG spectra of the vapor/ice (0001) interface at various temperatures. The polarization combination is $s s p$. 


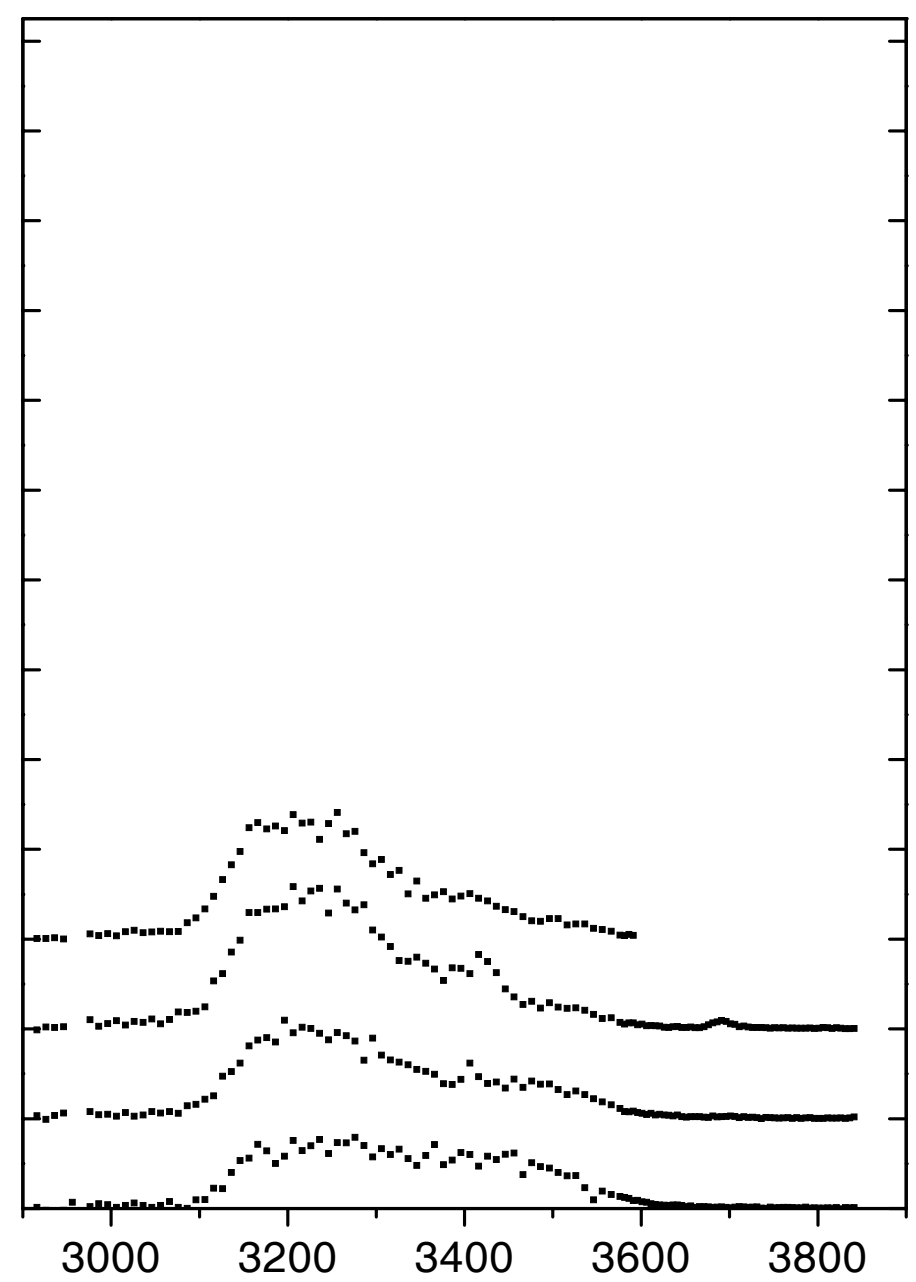

